\title{
CONVERGENCIAS ESTRATÉGICAS ENTRE LAS HUMANIDADES Y LAS CIENCIAS DE LA INFORMACIÓN. UNA OPORTUNIDAD PARA LA TRANSFORMACIÓN DIGITAL EN CUBA
}

\author{
Sulema Rodríguez-Roche ${ }^{1}$ \\ Universidad de la Habana, Cuba \\ sulema1985@gmail.com
}

Ania R. Hernández-Quintana ${ }^{2}$

Universidad de la Habana, Cuba aniahernandez.quintana@gmail.com

\begin{abstract}
Resumo
El trabajo aborda la emergencia de las Humanidades Digitales y justifica los principales ejes en que confluye con los valores y prácticas de las Ciencias de la Información. Se presenta el contexto académico y profesional de las Ciencias de la Información en Cuba como escenario para entronizar los principales contenidos que caracterizan la enseñanza y producción de las comunidades de humanistas digitales. Desde el análisis del estado de las relaciones interdisciplinares y de los objetivos y metas de la agenda 2030, especialmente lo relacionado con el acceso pleno a la información, se propone una mirada transversal, adaptada a la realidad nacional, para intervenir en el actual y relativo distanciamiento entre los campos de las Humanidades Digitales y las Ciencias de la Información. Esta investigación concluye la primera etapa de desarrollo del Grupo de investigación académica Humanidades Digitales y Ciencias de la Información (HDCICuba), proyecto institucional de la Facultad de Comunicación de la Universidad de La Habana.
\end{abstract}

Palavras-chave: Transversalización; Humanidades Digitales; Ciencias de la Información; HDCICuba

\begin{abstract}
Abstract

'New media' information technologies were recently thought to be so intrinsically different from 'old,' mass media, technologies that fascism would no longer be possible. Through new media information and communication technologies, the political 'mass' was supposedly replaced by the 'crowd' or the 'swarm,' and an old mass media replaced by a new media serving individual 'information needs.' However, extreme right-wing political populism and encroaching fascism today are world-wide phenomena in developed countries, not only despite new media, but partly because of it. How is this possible?
\end{abstract}

Keywords: Transversalization; Digital Humanities; Information Sciences; HDCICuba

\footnotetext{
${ }^{1}$ Profesora Asistente de la Facultad de Comunicación de la Universidad de la Habana. Candidata a Doctora en Ciencias de la Información. Investigadora asociada al Laboratorio de Innovación de Humanidades Digitales (LINHD), de la UNED, España. sulema1985@gmail.com

2 Profesora Titular del Departamento de Ciencias de la Información de la Facultad de Comunicación de la Universidad de la Habana. Doctora en Ciencias de la Información. Líder del grupo de investigación académica Humanidades Digitales y Ciencias de la Información en Cuba (HDCICuba). Vicepresidenta de la Sociedad Cubana de Ciencias de la Información (SOCICT).
}

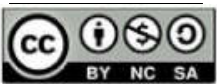

Esta obra está licenciada sob uma Licença Creative Commons Atribuição 4.0 Internacional (CC BY-NC-AS 4.0). LOGEION: Filosofia da informação, Rio de Janeiro, v. 6 n. 1, p.186-208, set.2019/fev. 2020 


\section{INTRODUCCIÓN}

El final del siglo XX constituye una vorágine de transformaciones, entre otras razones, por la asunción exponencial, cotidiana y casi transparente de continuas revoluciones tecnológicas directamente vinculadas con las formas de transmisión de la información, fenómeno sólo comparable con el cambio cultural que significó la invención de la imprenta.

Consecuentemente, en la década del 90, comenzaron los estudios para conocer cómo la sociedad enfrentaría los desafíos de la informatización en la esfera de los registros organizados de la memoria social (Dodebei, 2011). Para ello, debían ser revisados los procedimientos teóricos, conceptuales y metodológicos de cada una de las disciplinas tradicionales que tenían a la memoria como objeto de estudio. En esos momentos ya había suficientes argumentos sobre la importancia de los estudios informacionales para la sobrevivencia no sólo de las instituciones sino de los medios de circulación de los bienes culturales, entre los cuales se encuentran las bibliotecas, los archivos y los museos.

La información disponible en la red en el siglo XXI y la multiplicidad de artefactos asociados, impone a las Humanidades y Ciencias Sociales un amplio y complejo escenario de pesquisas sobre el homo digital y sus diferentes roles en la transformación digital de las sociedades.

$\mathrm{Si}$ las dinámicas tecnológicas están modificando los modos de construcción e institucionalización de los saberes, aquellos que emergen del campo de las Ciencias de la Información debieran, de forma mucho más natural, no sólo coexistir sino establecer una suerte de cohesión profunda para rediseñar sus propuestas metodológicas y tradiciones profesionales.

Los abordajes filosóficos sobre las Ciencias de la Información que encabezaran Shera y Borko en el siglo XX, producto de las cambiantes dinámicas que también desde lo tecnológico impactaban al campo, se reactivan en el siglo XXI con las consideraciones del filósofo italiano Luciano Floridi (2002), oriundo del área computacional, en su intento de sustituir la epistemología social del conocimiento por una filosofía de la información aplicada, la cual acarreó toda suerte de discusiones, a favor y en contra.

Por el contrario, los presupuestos de los sociólogos Gilles Deleuze y Félix Guattari han sido más reconocidos a la hora de verificar cómo los dividendos de la relación postmodernidadtecnologías se evidencian desde el pensamiento humanista en el campo informacional. Estos autores llegaron al punto de reconfigurar casi todas las áreas de las Ciencias de la Información desde el concepto de "rizoma", en clara alusión al impacto de la red de redes. La propuesta rizomática ayuda lo mismo a desarrollar el conjunto de análisis sobre el ciberespacio y los 
aspectos filosóficos de lo virtual, como el acercamiento a las Ciencias de la Información desde su multidisciplinariedad y campo múltiple de actuación. (Day, 2010)

Siendo las ciencias y las tecnologías producciones culturales, deberían percibirse también desde lo que puedan nutrirse, servirse, interpretarse y combinarse (Vinck, 2013). La posibilidad de copiar, pegar, anotar o hiperligar, convierte a la digitalización en otro "móvil inmutable" distinto de los libros, al decir de Latour (1992), en un objeto de reflexión epistémica y operativa de las Ciencias de la Información.

Para ese nuevo (otro) objeto, las Ciencias de la Información son un escenario de codificación y decodificación cultural, donde importa estudiar el proceso de conservación de sus datos, sus formas de articulación en redes o sus programas de organización y tratamiento. Al manipular directamente elementos de la sociedad, como su patrimonio, su identidad o sus representaciones, la digitalización se convierte en una forma de traducción de esos elementos, marca una elección e impulsa la transformación digital.

Las infraestructuras digitales llegaron primero a la Bibliotecología que a cualquier otra disciplina humanística y social. El hito es marcado por las primeras experiencias de automatización de bibliotecas. Desde entonces, los profesionales de las Ciencias de la Información, involucrados en procesos digitales y movilizados desde sus presupuestos, requieren reconstruir sistemáticamente la experiencia de formación, de investigación y de quehacer profesional para aprender, usar y crear nuevos sistemas de representación y acceso.

Casi 50 años después, continúa siendo preciso un profesional capaz de lidiar con información independientemente de su formato, cada vez más consciente de las implicaciones sociales, de las modificaciones de los procesos de comunicación y de la adaptación de los métodos de organización intelectual.

A las Humanidades Digitales le interesa la investigación digital de la cultura, la gestión de datos humanísticos e incrementar desde ellos la comprensión cultural del pasado y de lo emergente. En sentido estricto, pero lamentablemente, esto no garantiza automáticamente la democratización de los conocimientos. Detrás de toda novedad persisten escenarios de hegemonías diversas, y en este caso, los poseedores de los medios de representación, digitalización, almacenamiento y acceso a información digitalizada participan también de estrategias geopolíticas de dominación que son reales y que convenientemente deben ser estudiadas, sin ingenuidad.

Las Humanidades Digitales son una tendencia en los programas de formación e investigación en las universidades, en tanto responden a potenciales conflictos en la relación entre cultura y tecnología. Las escuelas de Ciencias de la Información en varios países y sus 
redes profesionales comienzan a vincular los enfoques humanistas digitales desde la comprensión de múltiples escenarios de confluencia. Sin embargo, Cuba no posee una estrategia articulada académicamente para asumir los derroteros de las Humanidades Digitales.

El análisis de este tema permite obtener, en el orden teórico, una concepción sobre la confluencia de las Humanidades Digitales en el campo de las Ciencias de la Información. En esa dirección, el presente trabajo tiene como objetivo: Identificar las convergencias estratégicas entre las Humanidades Digitales y las Ciencias de la Información.

\section{METODOLOGÍA}

La presente investigación tiene un enfoque mixto, pues combina el uso de métodos y técnicas cualitativas y cuantitativas. Se apoya en la E-Investigación (E-Research) y el Análisis Exploratorio de Datos (DEA). El primero es un paradigma de la investigación científica en las Ciencias Sociales y Humanas que incorpora el uso intensivo de las Tecnologías de Información y Comunicación (TIC) por las facilidades que ofrecen para la investigación en colaboración. El segundo es una filosofía de análisis de información que consiste en resumir las características principales de la información de una manera fácil de comprender, usualmente explotando visualizaciones gráficas, sin la utilización de modelos estadísticos concretos.

\subsection{MÉTODOS Y TÉCNICAS}

Análisis de contenido (AC): Parte del principio de que examinando textos es posible conocer su significado y obtener información respecto a su modo de producción. Es decir, trata a los textos no sólo como signos dotados de un significado conocido por su emisor, sino como indicios del emisor y del modo de producción de un texto. Este método permitió analizar y corroborar las categorías teóricas y metodológicas y además permitió elaborar los análisis bibliográficos.

Análisis de Componentes Principales (ACP): Técnica estadística de reducción de la dimensionalidad que se utiliza para describir un conjunto de datos presumiblemente dependientes con el fin de crear variables (componentes) no correlacionadas. Asumiendo que las Humanidades Digitales y las Ciencias de la Información están relacionadas, el ACP permitió visualizar y jerarquizar esa relación. Aplicado sólo a las Humanidades Digitales, permitió obtener la información necesaria para crear los ejes transversales a las Ciencias de la Información. 
Análisis de Redes Sociales (ARS): Método que permite describir y visualizar los actores y sus relaciones para un contexto determinado y que ayuda a comprender los entornos de influencia en la red. En esta investigación se usó para analizar la estructura de las relaciones entre Humanidades Digitales y Ciencias de la Información; así como de las categorías de las Humanidades Digitales que se encontraban en el mismo componente (ACP), con las categorías de Ciencias de la Información para obtener los contenidos transversales.

UCINET: Paquete de software propietario para el análisis de datos de redes sociales, desarrollado por Lin Freeman, Martin Everett y Steve Borgatti, en Harvard University. ${ }^{3}$

Netdraw: Módulo de UCINET que permite graficar redes sociales y obtener matrices.

EndNote: Paquete informático de gestión de referencias y citas bibliográficas, propiedad de Thomson Reuters. ${ }^{4}$ La biblioteca que se adjunta contiene los textos utilizados para el análisis cienciométrico, útil para estudiar las predisposiciones de las Ciencias de la Información y las convergencias con las Humanidades Digitales.

Se utilizaron las siguientes herramientas para la obtención y procesamiento de la información:

Matlab: De MATrix LABoratory, "laboratorio de matrices", es un sistema de cómputo numérico que ofrece un entorno de desarrollo integrado (IDE) con un lenguaje de programación propio (lenguaje M). Es posible ampliar sus capacidades con cajas de herramientas (toolboxes), entre las que se destacan varias para el análisis estadístico que fueron muy útiles en la investigación.

VOSviewer: Herramienta de escritorio para la construcción y visualización de redes bibliométricas. Permitió caracterizar las convergencias entre Humanidades Digitales y Ciencias de la Información por la coocurrencia de categorías y palabras clave.

\subsection{PREPARACIÓN Y ESTRUCTURACIÓN DEL CORPUS}

Para obtener el corpus se cumplimentó el siguiente protocolo de búsqueda y recuperación:

1. Se realizó una búsqueda libre de la producción científica sobre Humanidades Digitales y Ciencias de la Información en las bases de datos EBSCO y WOS, entre 2008 y 2018.

\footnotetext{
${ }^{3}$ Aún cuando hubiera sido deseable utilizar exclusivamente herramientas de código abierto para ser coherente con uno de los valores de las Humanidades Digitales, las dificultades de acceso y la experticia del entorno condicionó su selección.

${ }^{4}$ Se utilizó para poder exportar en el formato seleccionado.
} 
2. Se realizó una búsqueda en Google para incluir la producción científica fuera de la corriente principal.

3. Se utilizaron los siguientes términos, en español e inglés, como estrategias de búsqueda: Humanidades Digitales y Ciencias de la Información, Digital Humanities and Information Sciences, Humanidades Digitales y Bibliotecología, Digital Humanities and Librarianship, Humanidades Digitales y Archivística, Digital Humanities and Archival Studies, Humanidades Digitales y Ciencia de la Información, Digital Humanities and Information Science.

Como resultado de la búsqueda se recuperaron 682 registros, de los que resultaron pertinentes para la investigación 241 (35,3\% del total). Los registros de la muestra longitudinal es lo que se denomina corpus en esta investigación, como un ejercicio de adaptación a la terminología de las Humanidades Digitales.

El corpus se organizó en una biblioteca EndNote, donde cada registro fue categorizado (variables) e indizado (observaciones). Las categorías se conformaron con las siglas de los campos (HD y CI), un guión (-) y los componentes que Fuentes (1997) recomienda para el estudio del campo académico (Teoría, Investigación, Formación y Profesión). De esta forma, se obtuvieron ocho variables aleatorias, a saber: HD-Teoría, CI-Teoría, HD-Investigación, CIInvestigación, HD-Formación, CI-Formación, HD-Profesión, CI-Profesión. La indización se realizó por asignación de palabras clave. Se obtuvieron 374 términos que sirvieron como observaciones para el análisis y discusión del corpus (HD: 225, CI: 149)

\section{RESULTADOS}

Con el objetivo de caracterizar estadísticamente las relaciones entre Humanidades Digitales y Ciencias de la Información, se procesó el corpus con un Análisis de Componentes Principales (ACP) y un Análisis de Redes Sociales (ARS), y finalmente un Análisis de Contenido (AC).

\subsection{ANÁLISIS DE COMPONENTES PRINCIPALES}

La interrelación entre Humanidades Digitales y Ciencias de la Información a nivel de palabras clave se representa en la matriz de correlación cruzada de las variables aleatorias que corresponde a la Tabla 1. Se consideraron un total de 30 palabras clave, cuya frecuencia de aparición fue $=>10$. Las autocorrelaciones significativas se muestran en negrita.

Las correlaciones se establecieron entre la mayoría de las variables de los dos grupos disciplinarios. La autocorrelación más significativa se presenta entre HD-Investigación y CI- 
Investigación, seguido de HD-Formación y CI Formación. En general, se demuestra que existe relación pero no solapamiento entre los campos de Humanidades Digitales y Ciencias de la Información, lo cual demuestra las zonas de convergencia y también las especificidades de ambos campos.

Tabla 1: Matriz de autocorrelación de las variables aleatorias

\begin{tabular}{|l|l|l|l|l|l|l|l|}
\hline & Cl-Teoría & Cl-Investigación & Cl-Formación & Cl-Profesión & HD-Teoría & $\begin{array}{l}\text { HD- } \\
\text { Investigación }\end{array}$ & HD-Formación \\
\hline Cl-Teoría & 1 & & & & & & \\
\hline Cl-Investigación & 0.0572725 & 1 & & & & & \\
\hline Cl-Formación & -0.0672595 & -0.0214615 & 1 & & & & \\
\hline Cl-Profesión & $\mathbf{0 . 4 7 1 0 6 5 7 1}$ & 0.26776736 & 0.02672026 & 1 & & & \\
\hline HD-Teoría & 0.37828008 & 0.16674688 & 0.04153271 & $\mathbf{0 . 4 2 3 8 6 7 6 1}$ & 1 & & \\
\hline HD-Investigación & -0.1384874 & $\mathbf{0 . 7 2 4 7 0 5 5 3}$ & -0.2065174 & 0.21102642 & $\mathbf{0 . 4 0 9 7 4 0 5 7}$ & 1 & \\
\hline HD-Formación & -0.1715477 & -0.0098618 & $\mathbf{0 . 5 8 9 0 2 6 9 9}$ & -0.1461824 & 0.2849067 & 0.00539698 & 1 \\
\hline HD-Profesión & -0.020748 & -0.0620227 & 0.04411202 & 0.20673927 & 0.57351644 & 0.2610334 & 0.26133661 \\
\hline
\end{tabular}

Figura 1: Proyección de las variables aleatorias obtenidas del Análisis de Componentes Principales.

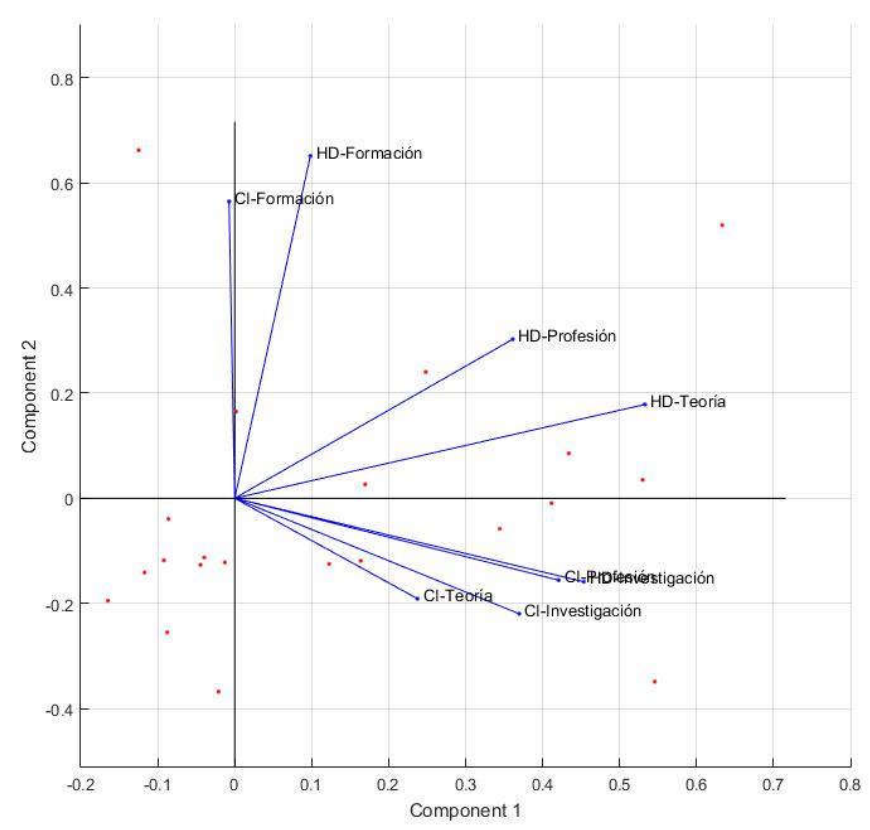

Como resultado de la aplicación del ACP se obtuvo la proyección de las variables aleatorias originales en los tres componentes principales, como se observa en la Figura 3.

Se obtuvieron dos grupos de variables: El grupo de CI-Teoría, CI-Investigación, CIProfesión, el grupo HD-Teoría, HD-Investigación, HD-Profesión. A este grupo se le identificó con el nombre Investigación por ser la categoría de mayor proyección hacia la componente principal. 
El grupo HD-Formación y CI-Formación tiene muy baja proyección en el componente principal y conforma una componente independiente casi exclusiva. A este grupo se le identificó con el nombre Formación.

La aplicación del ACP permitió reducir la dimensionalidad de las relaciones entre las Humanidades y las Ciencias de la Información y demostrar que efectivamente existen confluencias en el orden teórico e investigativo, en primer lugar y en el orden profesional y formativo, en segundo lugar.

\subsection{Análisis de Redes Sociales}

La asociación y medida de las relaciones y flujos entre las Humanidades Digitales y las Ciencias de la Información, demuestra la consistencia de los resultados del ACP.

Figura 2: Correlación de categorías por componente.
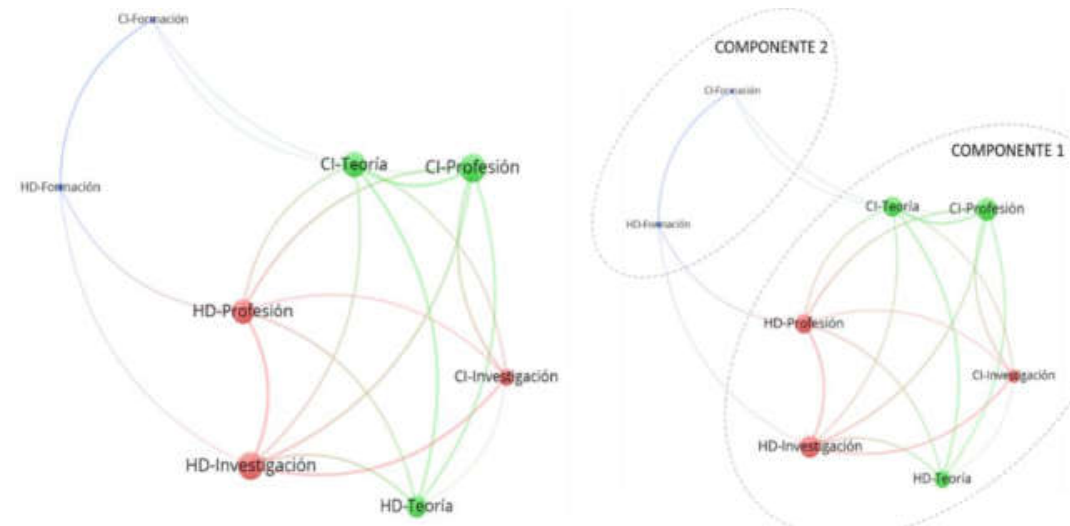

Siguiendo la lógica de las explicaciones del ACP, se describen primero las del componente principal y luego las del componente 2. Las categorías HD-Profesión, HDInvestigación, CI-Investigación (conectadas en color rojo), representan las relaciones más fuertes, creando un entramado con las categorías CI-Teoría, HD-Teoría, CI-Profesión (representadas en color verde) y muestran las relaciones promedio de correlación de términos.

En comparación con la formación de subgrupos obtenidos del ACP, el entramado de las categorías representadas en verde y rojo, refieren conexiones múltiples entre todos los nodos, lo cual es consiste con la estructura del componente principal. Las relaciones más débiles (coloreadas en azul), constituyen una familia compuesta por las categorías HD-Formación y CI-Formación, consistente con el componente 2. 
La categoría HD-Investigación se conecta con todas las categorías, excepto con CIFormación. Esto sucede porque sólo en cuatro registros coinciden las categorías HDInvestigación y CI-Formación, lo cual no es significativo.

La relación entre los términos de cada nodo con el resto, visualiza baja correlación de los temas de formación en ambos grupos, respecto al resto de las categorías. La tendencia a fortalecer la relación de CI-Formación con CI-Profesión y CI-Teoría es consistente con la estructura del campo, si bien los datos no muestran relación con la categoría CI-Investigación. CI-Formación es un nodo muy débil en la correlación, porque en este corpus no existen registros que vinculen a los planes de estudio de Humanidades Digitales y Ciencias de la Información.

Por otra parte, se observa la relación entre HD-Formación, con las categorías HDProfesión y HD-Investigación, a la vez que no se conectada al nodo HD-Teoría. Esto se constata con la mínima cantidad de registros, solamente cuatro, que contienen términos relacionados a HD-Formación y HD-Teoría, que no son representativos para el software utilizado.

La coocurrencia de las palabras clave y categorías en una red de modo 2 se presentan en la Figura 6. Se aprecia que las categorías más fuertemente relacionadas son HDInvestigación y CI-Investigación. Esto reafirma que la investigación es el cuerpo estructurante de las Humanidades Digitales y que la investigación en Ciencias de la Información se presenta como un síntoma de la fortaleza del campo. El estado de cercanía y verticalidad entre estos nodos consolida una importante zona de convergencia entre los campos y sirve de argumento para que en la propuesta se enuncien a través de la investigación un número significativo de acciones de transversalización.

Se aprecia una superposición entre las categorías CI-Formación y CI-Profesión. Esto denota una fortaleza del campo y permitirá enunciar en la propuesta que los escenarios más aptos para la transversalización se encuentran en esas dimensiones.

El nodo de la categoría HD-Investigación es el de mayor número de términos asociados. Las categorías HD-Teoría, CI-Teoría, CI-Investigación, CI-Profesión y HD-Formación tienen una relación de términos significativa; mientras que las categorías HD-Profesión y CIFormación tienen menos términos conectados.

Los términos que se encuentran inmersos entre esas categorías son los de mayor confluencia e interés para la formulación de la propuesta. Atendiendo a la visibilidad de los términos, por ejemplo, a través del uso de las TIC, los proyectos relacionados con bibliotecas universitarias y, en general, temas de preservación digital deberán ser potenciados. Así también, la evidente fortaleza de las redes de Humanidades Digitales a nivel internacional deberá servir de precedente para fortalecer este tipo de estructura en Cuba. Deberá destacarse también en la 
propuesta, la atención al tratamiento de los datos, que es otro de los términos que destaca en esta zona.

De la relación entre HD-Teoría y CI-Teoría se construyen las siguientes inferencias: La primera apunta a que el número de registros que reflejan el interés por comunicar las áreas de interés de las Humanidades Digitales no sólo es numeroso sino coherente con sus postulados epistémicos. La segunda, que el discurso teórico de las Ciencias de la Información está cada vez más comprometido con la transdisciplinariedad. La tercera y principal, que teóricamente son campos con basamentos distintos y diferenciables pero que coinciden a través de las relaciones disciplinares.

Figura 3: Red de coocurrencia de categorías y palabras clave.

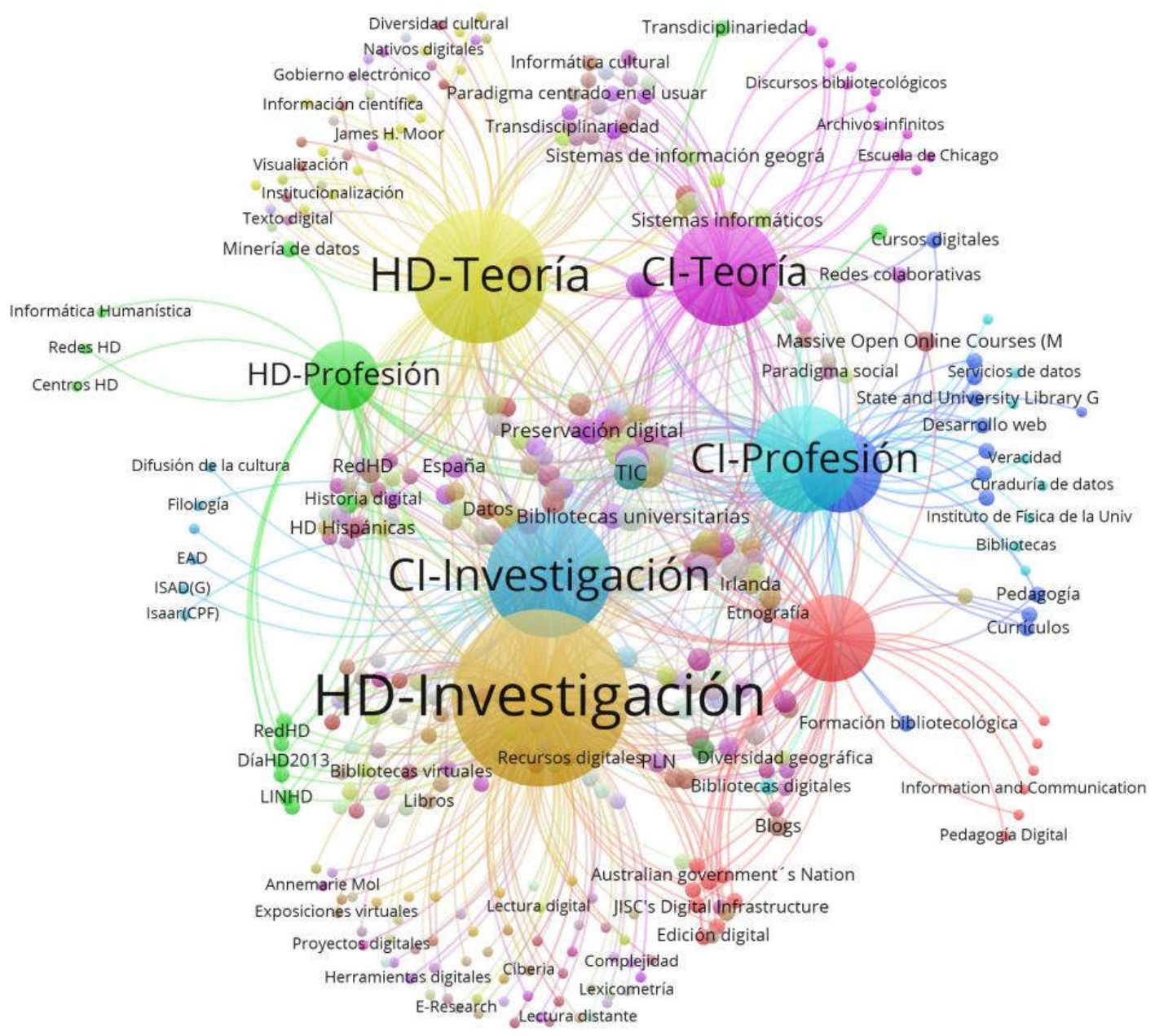

En la correlación entre categorías y palabras clave se muestran como términos consolidados las categorías: HD-Investigación, HD-Teoría, CI-Investigación, CI-Profesión, CI- 
Teoría, en ese orden, y las palabras clave: TIC y Humanidades. El mapa demuestra el peso, otra vez, de la investigación en Humanidades Digitales y el escenario donde actúa. También aparece CI-Profesión como categoría consolidada. Si bien esta imagen bidimensional no lo representa, en la imagen ampliada CI-Formación aparece como un escenario consolidado solamente en la Bibliotecología.

En la zona emergente, los términos a destacar son las categorías HD-Profesión y HDFormación y las palabras clave Bibliotecas Universitarias, Infraestructuras tecnológicas y Metadatos. Formación Esto puede ser considerado en la propuesta para enunciar a los humanistas digitales como salida o perfil profesional del programa de Ciencias de la Información y como tipología para la cual deben prepararse los distintos escenarios del campo profesional.

Las relaciones más débiles entre las categorías y las palabras clave apuntan a entidades, sistemas y herramientas. Se evidencian términos que sólo se relacionan con una categoría, como es el caso de Edición digital. Si bien es un tema concurrente para ambos campos, este proceso aparece como una fortaleza de las Humanidades Digitales más que de las Ciencias de la Información.

En la proyección de la densidad de las categorías y palabras clave se encontró que los términos ubicados en la zona de mayor densidad son: TIC (46), Colaboración (42), Proyectos digitales (41), Cultura digital (40), E-Research (28), Herramientas digitales (28) Bibliotecas (26), Centros HD (26), Bibliotecología (23).

Los términos bibliotecas, colaboración, herramientas digitales, proyectos digitales y TIC, que conforman el núcleo de densidad en la zona consolidada, representan los componentes de casi todos los emprendimientos en Humanidades Digitales. En la literatura analizada se refiere el carácter colaborativo de estos, así como el uso de herramientas tecnológicas para la ejecución de proyectos, que pueden tener como salida una biblioteca. La E-Research, es el paradigma de investigación desde el cual se ejecutan esos proyectos. Los centros de Humanidades Digitales son el espacio en el que se producen esas investigaciones.

La lectura de la densidad de palabras clave permite concluir que la relación entre Humanidades Digitales y Ciencias de la Información está consolidada en las bibliotecas y que las salidas más frecuentes son los proyectos de Humanidades Digitales. 


\subsection{ANÁLISIS DE CONTENIDO}

Ramsay (2010) formula su tesis sobre las convergencias de las Humanidades Digitales con las Ciencias de la Información, en base a una de las funciones identitarias del escenario informacional, la organización del conocimiento:

De todos los objetivos académicos, Humanidades Digitales es la que más claro representa el espíritu que animó la antigua fundación de Alejandría, Pérgamo y Memphis, las grandes bibliotecas monásticas de la Edad Media, e incluso las primeras bibliotecas de la Ilustración Alemana. Está obsesionada con variedades de representaciones, organización del conocimiento, las tecnologías de la comunicación y la diseminación, así como la producción de herramientas útiles para uso académico.

Sula (2013) reportó un incremento sostenido de las publicaciones sobre Humanidades Digitales en LISTA y que esas apariciones se duplicaron en 2012.

A pesar de las coincidencias entre las Ciencias de la Información y las Humanidades Digitales, y del creciente número de proyectos de Humanidades Digitales que involucra a especialistas en información, en la literatura consultada demuestra que no resulta fácil iniciar y sostener esas colaboraciones por varias razones. Primero porque los académicos humanistas, entrenados de forma tradicional, se encuentran estereotipados por sus actividades de investigación autónoma (Spiro, 2011), por lo que se necesitan esfuerzos mayores de los profesionales de la información para establecer un diálogo de confianza. Segundo, como campo en su fase embrionaria, en Humanidades Digitales no se sabe con certeza cómo garantizar proyectos exitosos con un impacto duradero (Zhang et al., 2015).

En el orden conceptual, se registran en la literatura definiciones de Humanidades Digitales en la intersección de las nociones de investigación, tecnología, y bibliotecas. En principio, las Humanidades Digitales colaboran con las bibliotecas en la alineación de sus servicios de soporte e infraestructura a la e-investigación; a la vez que sirven como agente de agente de cambio en la redefinición de enfoques, proyectos, roles profesionales y relaciones entre academias, centros e investigadores.

El número 1, del volumen 53 de la Journal of Library Administration es paradigmático en este sentido, pues reunió los trabajos de Vandegrift \& Varner; Sula y Posner respecto a las tres dimensiones consideradas territorios de convergencia de las Humanidades Digitales y la Bibliotecología: la biblioteca como infraestructura de investigación (Vandegrift \& Varner, 2013), la cercanía conceptual entre Humanidades Digitales y Bibliotecas (Sula, 2013) y las 
competencias profesionales requeridas por los bibliotecarios en Humanidades Digitales (Posner, 2013).

En sus respectivas contribuciones, los autores separan humanidades y bibliotecas; no porque bibliotecas y Bibliotecología (Librarianship es el punto de observación) no sea también humanidades, aparentemente, sino porque deben distinguir el foco de atención (biblioteca y Bibliotecología) del resto de las disciplinas de Humanidades.

\subsubsection{La biblioteca como infraestructura de investigación}

Vandegrift \& Varner (2013) discutieron cómo las tecnologías hacen más accesible y comprometido el trabajo de humanistas digitales y bibliotecarios. Comentaron que el movimiento de acceso abierto y el deseo de hacer más relevante la investigación para el mundo, más allá de la academia, hace que las bibliotecas "reinventen sus espacios en el ciclo de producción académico".

Sula (2013) explicó las condiciones de posibilidad que ofrece la Bibliotecología a las Humanidades Digitales. Considerando a las Humanidades Digitales como una tendencia de investigación en Humanidades, el autor argumenta la naturaleza cambiante de la Bibliotecología, sitúa a los bibliotecarios en posición de ofrecerle soporte. Para sustentar su postura, mapea las competencias en Humanidades Digitales y las relaciona con las de la American Library Association Core Competiences in Librarianship, encontrando varias coincidencias: recursos de información digitales, organización del conocimiento, conocimiento y habilidades tecnológicas y servicios a usuarios.

El estudio documental de Sula (2013) mostró que, en el espacio estadounidense, el trabajo de los humanistas digitales es diverso y sus colaboraciones con las bibliotecas es idiosincrático. Formula, en consecuencia, el modelo de Humanidades Digitales y Bibliotecas basado en el enfoque de la Informática cultural (Cultural Informatics), la cual se interesa por el impacto cultural de las tecnologías de la información.

El modelo, se refiere a la "multiplicidad de formas en las que pueden apoyarse, comprometerse y crear, de forma conjunta, las bibliotecas y las Humanidades Digitales" (Sula, 2013). Se representa en cuadrantes interceptados en el eje de las Y por la relación humano (human)-computadora (computer) y en el de las X por las “órdenes de contenido", a la izquierda el contenido de primer orden (first-order content) y a la derecha el contenido de segundo (second-order content): 
La superposición del trabajo del contenido de primer orden - segundo orden, y del poder humano-computadora, sugiere que bibliotecas y Humanidades Digitales están en efecto, involucradas en actividades complementarias (...) y las Humanidades Digitales tienen un papel imperecedero dentro del mundo de las bibliotecas.

\subsubsection{Los bibliotecarios en Humanidades Digitales}

Gibson, Ladd \& Presnell (2015) explicaron, en términos de proyectos, las implicaciones de los contenidos de primer orden y de los contenidos de segundo orden. Señalaron que los de primer orden son aquellos que corresponde a recreaciones digitales de materiales ya existentes, como colecciones digitales de cartas, con poco o ningún análisis incluido; los de segundo orden toman materiales digitales y los mejoran, usando cualquiera de la gran variedad de herramientas y técnicas digitales, para comprender/responder completamente una pregunta de investigación, como por ejemplo mapear dónde fue escrita una carta para comprender el contexto geográfico en la cual fue escrita o correlacionar tasas de alfabetización con localizaciones de bibliotecas y librerías. Un aspecto interesante en este trabajo es el análisis transversal de las funciones del bibliotecario especializado (subject librarian), que denominan Transversing the gap.

La "brecha" se encuentra en la incorporación de bibliotecarios a los proyectos, en los roles que estos asumen o le son asignadas, así como las competencias y habilidades profesionales fundamentalmente en el manejo de herramientas tecnológicas que los bibliotecarios requieren y no siempre poseen. Es un hecho que bibliotecarios y programadores tienen que trabajar de forma conjunta. (Gibson, Ladd \& Presnell, 2015)

La convergencia entre Humanidades Digitales y Bibliotecología se explica en la naturaleza colaborativa de los proyectos de humanidades digitales. Los autores señalaron que, como regla general, esos proyectos incluyen roles para académicos, tecnólogos y bibliotecarios especializados.

Académicos, tecnólogos y bibliotecarios asignados de humanidades traen cada uno un enfoque único: el académico, el conocimiento del contenido; el tecnólogo, las habilidades tecnológicas necesarias; y el bibliotecario especializado, la comprensión abarcadora de la investigación de humanidades digitales (Gibson et al., 2015).

La American Library Association (ALA), publicó desde 2009 sus Core Competencies of Librarianship ${ }^{5}$, un repertorio de conocimientos considerados necesarios y que deben poseer

\footnotetext{
${ }^{5}$ http://www.ala.org/educationcareers/careers/corecomp/corecompetences
} 
los graduados de programas de maestría en Bibliotecología y Estudios de la información acreditados por este organismo.

Al revisar esas competencias, Bello, Dickerson, Hogarth \& Sanders (2017) hallaron cercanías respecto a las Humanidades Digitales en cuanto a:

- Conceptos y temas relacionados con el ciclo de vida del conocimiento registrado y la información, desde la creación hasta sus etapas de uso.

- Conceptos, problemas y métodos relacionados con la adquisición y disposición de los recursos, incluida la evaluación, selección, compra, procesamiento, almacenamiento y descarte.

- Conceptos, problemas y métodos relacionados con el mantenimiento de colecciones, incluyendo la preservación y conservación.

- Las habilidades de desarrollo descriptivas y de evaluación necesarias para organizar los recursos del conocimiento y la información registrada.

- Manejo de sistemas de catalogación, metadatos, indización y normas de clasificación y métodos utilizados para organizar el conocimiento y la información registrada.

- Los principios y las técnicas necesarias para identificar y analizar las tecnologías e innovaciones emergentes con el fin de reconocer e implementar mejoras tecnológicas pertinentes.

- Técnicas de competencia de la información, métodos de alfabetización informativa y de cultura estadística.

- Los principios y métodos de promoción que se utilizan para llegar a audiencias específicas para promover y explicar conceptos y servicios.

- $\quad$ Principios de evaluación y respuesta a la diversidad de necesidades de los usuarios, comunidades de usuarios y las preferencias del usuario.

- Principios y métodos utilizados para evaluar el impacto de las situaciones o circunstancias actuales y emergentes en el diseño e implementación de servicios apropiados o desarrollo de recursos.

- Los fundamentos de los métodos de investigación cuantitativa y cualitativa.

- La necesidad de continuar el desarrollo profesional de los profesionales de las bibliotecas y otras agencias de información. 
Posner (2013) refirió los retos de "hacer" Humanidades Digitales en las bibliotecas, e identificó un grupo de competencias necesarias a las que denominó "soft skills "6, tales como: gestión de proyectos, análisis del contexto y promoción de las relaciones con los académicos.

Zhang, et al. (2015) realizaron un estudio, con datos de Centernet, que tuvo como objetivo analizar las convergencias entre las Bibliotecas Digitales (Digital Libraries-DL) y las Humanidades Digitales (Digital Humanities-DH).

Los autores refirieron que las bibliotecarios académicos cada vez más tienden a colaborar con las facultades de Humanidades Digitales; aparecen entre los resultados la creación de infraestructuras regionales como la europea y de proyectos específicos, como el denominado The Developing Librarians, de la Universidad de Columbia, un programa de dos años para formar a los bibliotecarios en las habilidades y metodologías de las Humanidades Digitales.

Analizaron además la profesión bibliotecaria en la dimensión histórica y en las prácticas más recientes, para establecer una serie de Do's and Dont's ${ }^{7}$ de la profesión para el trabajo en Humanidades Digitales. Estas se resumen en la siguiente tabla:

Tabla 2: Experiencia y nuevos roles del bibliotecario para el trabajo en Humanidades Digitales.

\begin{tabular}{|c|c|}
\hline Experiencia (Do's) & Nuevos roles (Dont's) \\
\hline $\begin{array}{l}\text { Proveedor o creador de contenidos que identifica y } \\
\text { desarrolla grandes (en tamaño) y diversas (en formato } \\
\text { y tema) colecciones digitales que están disponibles de } \\
\text { forma abierta para uso y reúso. }\end{array}$ & $\begin{array}{l}\text { Mediador e intérprete que posee la experticia tanto en } \\
\text { conocimiento e información como en tecnología. }\end{array}$ \\
\hline $\begin{array}{l}\text { Curador que evalúa, selecciona, organiza, y preserva } \\
\text { colecciones digitales, proyectos, materiales de } \\
\text { entrenamiento y más, asegurando su sostenibilidad y } \\
\text { acceso a largo plazo. }\end{array}$ & $\begin{array}{l}\text { Creador de espacios físicos y virtuales equipados con } \\
\text { tecnologías esenciales para el aprendizaje, } \\
\text { investigación, enseñanza y actividades en equipo de la } \\
\text { comunidad de Humanidades Digitales. }\end{array}$ \\
\hline $\begin{array}{l}\text { Mensajero o enlace que de forma proactiva encuentra } \\
\text { a demanda las colecciones digitales apropiadas, } \\
\text { herramientas, servicios y oportunidades de } \\
\text { financiamiento para sus usuarios potenciales. }\end{array}$ & $\begin{array}{l}\text { Aliado que provee servicios de soporte y está } \\
\text { comprometido con el desarrollo de nuevas } \\
\text { herramientas y recursos. }\end{array}$ \\
\hline $\begin{array}{l}\text { Educador e instructor que transfiere habilidades a los } \\
\text { estudiantes de Humanidades a través de talleres } \\
\text { prácticos (hands-on workshops) y consultas para } \\
\text { minimizar el malestar productivo (productive unease) }\end{array}$ & $\begin{array}{l}\text { Innovador que genera y comparte nuevas ideas } \\
\text { basadas en las investigaciones más recientes sobre } \\
\text { usuarios y estudios de la información; que } \\
\text { experimenta activamente con las interfaces, minería }\end{array}$ \\
\hline
\end{tabular}

\footnotetext{
${ }^{6}$ Puede ser traducido como competencias suaves.

${ }^{7}$ Puede ser traducido como lo hecho y lo no hecho
} 


\begin{tabular}{|l|l|}
\hline $\begin{array}{l}\text { cuando el estudiante interactúa con contenidos y } \\
\text { tecnologías digitales. }\end{array}$ & $\begin{array}{l}\text { de datos, visualización de datos y otros proyectos } \\
\text { innovativos; y quien crea infraestructuras sostenibles } \\
\text { para la investigación en Humanidades Digitales y la } \\
\text { comunicación académica. }\end{array}$ \\
\hline $\begin{array}{l}\text { Consultante que ofrece consejo profesional acerca del } \\
\text { derecho de autor, acceso abierto y temas relacionados. }\end{array}$ & $\begin{array}{l}\text { Académico híbrido, con experticia en Bibliotecología } \\
\text { Digitales. }\end{array}$ \\
\hline & $\begin{array}{l}\text { Comprometido con la promoción de la investigación } \\
\text { en Humanidades Digitales y la colaboración con } \\
\text { disciplinas periféricas, becarios de investigación de } \\
\text { humanidades y una comunidad mucho más amplia. }\end{array}$ \\
\hline
\end{tabular}

Damian, Almeida, Mello y Rodrígues (2015) registraron en Brasil un tímido avance en la incorporación de las Humanidades Digitales en las organizaciones de información. Su estudio incluyó un total de 24 sitios web relacionados con museos e instituciones culturales de la ciudad Ribeirão Preto, en el estado de São Paulo.

Los autores observaron, en los museos, un bueno uso del diálogo entre la mediación cultural, accesibilidad y tecnología, cuya generalización es un reto de los profesionales de la información en el ámbito de las Humanidades Digitales:

\footnotetext{
Sin embargo, llevar ese conocimiento y posibilidad de implementación a instituciones menores todavía parece ser una tarea difícil, así como un desafío para el profesional de la información, convirtiéndose en un área emergente para la actuación profesional, con la perspectiva de un mercado de trabajo creciente y con gran posibilidad de expansión. (Damian et al., 2015).
}

En la literatura analizada, se advirtió que las propuestas de colaboración entre las Humanidades Digitales y la Bibliotecología tienen como centro a la biblioteca, que puede funcionar como un espacio para los procesos de digitalización, procesamiento digital, preservación y servicios que caracterizan a los proyectos de Humanidades Digitales.

Algunas de esas posibilidades se han discutido en foros como el THATCamp Digital Library Federation Forum: Digital Humanities \& Libraries $^{8}$ y el THATCamp Digital Frontiers 9 . Estos eventos exploraron la creatividad y la colaboración a través de las fronteras disciplinarias (que sirven para unir, no separar) en el ámbito de las Humanidades Digitales y la memoria cultural, desde una variedad de perspectivas.

\footnotetext{
${ }^{8}$ http://dhlib2013.thatcamp.org/

9 http://digitalfrontiers2015.thatcamp.org/
} 


\subsubsection{La participación disciplinar}

Si bien existen evidencias de la relación de las Humanidades Digitales con las disciplinas informativas, ninguno de los modelos incluye de forma integrada a la Bibliotecología, la Archivística y la Ciencia de la Información. Por esta razón, se recomienda crear una infraestructura conceptual para la formalización esa relación como sustento a sus diálogos interdisciplinares.

Ortega y Gutiérrez (2014) estudiaron las interacciones de las disciplinas que comparten los intereses de las Humanidades Digitales. En una encuesta realizada a profesionales del MapaHD recabada en línea demostraron que, luego de la consabida penetración en los Estudios Literarios de las Humanidades Digitales, son las Ciencias de la Información el siguiente nodo significativo.

El análisis de intermediación permitió también advertir los vínculos entre Ciencias de la Información y otras disciplinas tales como Cine y Medios, Filosofía, Informática, Historia, Artes Visuales, Educación y Antropología. Las autoras explican este resultado en los planteamientos internos de esos campos respecto a las Humanidades Digitales:

\subsubsection{La propuesta de Koltay}

Tibor Koltay, jefe del departamento y director del currículo de Bibliotecología y Ciencia de la Información en el departamento de Information and Library Studies en Jászberény (Hungría), tiene una visión epistémica sobre el fenómeno que refiere todo este apartado. Koltay $(2016,2017)$ abordó los vínculos entre la Bibliotecología y Ciencia de la Información (BCI) ${ }^{10}$ y las Humanidades Digitales desde el objeto de estudio:

\footnotetext{
La mayor semejanza entre las dos disciplinas es su foco general en el estudio y la práctica relacionada con la información registrada, en otras palabras, documentos en un amplio sentido, que encapsula en "contenedores" todo el conocimiento registrado. Por lo tanto ambas, BCI y Humanidades Digitales pueden ser consideradas "ciencias de la información", en otras palabras, disciplinas interesadas en el estudio de la información.” (Koltay, 2016)
}

Esta perspectiva está sustentada en el concepto de la información como cosa ofrecido por Buckland (1991), que cubre cualquier cosa física que es percibida como relevante, y se

\footnotetext{
${ }^{10} \mathrm{El}$ autor se refiere a Library and Information Science (LIS)
} 
apoya en los trabajos de Schreibman et al.(2004), Alvarado (2012) y Robinson, Priego \& Bawden (2015).

Concebir la relación con las Humanidades Digitales desde el objeto de estudio simplifica y resuelve potenciales conflictos disciplinares, en tanto la postura inclusiva de Koltay no marca relaciones de poder ni dependencias a ultranza.

Por todo lo anterior, y para los fines de esta investigación, se entiende la relación convergente entre Humanidades Digitales y Ciencias de la Información desde el mapa de saberes y prácticas interdisciplinares comunes pero que no excluye las especificidades de cada una.

Los escenarios fundamentales de convergencia son el investigativo y el formativo. En el investigativo se encuentran los proyectos digitales, así como el conjunto de metodologías, métodos y herramientas para su realización. En el formativo los programas curriculares y extracurriculares de pregrado y posgrado, así como otras actividades, organizadas desde el escenario académico y profesional, tales como charlas, entrenamientos, talleres. La colaboración, el acceso abierto y la transparencia son valores distintivos y compartidos por las Humanidades Digitales y las Ciencias de la Información.

\section{CONCLUSIONES}

En el espacio digital se requieren competencias no resueltas por los lenguajes de programación y la arquitectura de software y sí desde las Ciencias Sociales y las Humanidades lo que ha llevado a su redescubrimiento en ese escenario. Las Humanidades Digitales ayudan a esa transformación, como un campo de conocimiento interdisciplinar y transversal, desde los diálogos que establece con las Ciencias de la Computación y con las Ciencias Sociales y las Humanidades.

La investigación y el aprendizaje constante forman parte de los compromisos fundacionales de las Humanidades Digitales, de ahí la necesidad de crear infraestructuras que faciliten la gestión de ese complejo proceso y retroalimenten sistemáticamente al sector profesional. Si bien existen centros y programas académicos de gran calidad, en las publicaciones científicas revisadas es más visible la formación a través de la investigación y la práctica profesional que la didáctica del proceso de formación.

Los escenarios fundamentales de convergencia son el investigativo y el formativo. En el investigativo se encuentran los proyectos digitales, así como el conjunto de metodologías, métodos y herramientas para su realización. En el formativo los programas curriculares y 
extracurriculares de pregrado y posgrado, así como otras actividades, organizadas desde el escenario académico y profesional, tales como charlas, entrenamientos, talleres. La colaboración, el acceso abierto y la transparencia son valores distintivos y compartidos por las Humanidades Digitales y las Ciencias de la Información. 


\section{REFERENCIAS}

ALVARADO, R. The digital humanities situation. In GOLD, M.K. (org) Debates in the Digital Humanities. Minneapolis/Londres: University of Minnesota Press 2012. Disponível em: http://www.arise.mae.usp.br/wp-content/uploads/2018/03/Debates-in-the-digitalhumanities-1.pdf . Consultado em:11 de setembro 2019.

BELLO, L.; DICKERSON, M.; HOGARTH, M.; SANDERS, A. Librarians Doing DH: A Team and Project-Based Approach to Digital Humanities in the Library. Collaborative Librarianship, V.9, $\mathrm{n}^{\mathrm{o}} .2$, 2017. P. 97-103. Disponível em:

https://digitalcommons.du.edu/cgi/viewcontent.cgi? article=1340\&context=collaborativelibrari anship Consultado em:11 de setembro 2019.

BUCKLAND, M. Information as thing. Journal of the American Society for Information Science, V.42. No.5. 1991. P. 351-360. Disponível em:

http://ppggoc.eci.ufmg.br/downloads/bibliografia/Buckland1991.pdf Consultado em:11 de setembro 2019.

DAMIAN, I.; ALMEIDA, M. A.; MELLO, T.; RODRIGUES, P. Convergências entre as Humanidades Digitais e a Ciência da Informaçao: o uso das TICs em unidades de informação. Ibersid, V.9. 2015. P.79-82. Disponível em: https://bdpi.usp.br/item/002733965 Consultado em:11 de setembro 2019. Consultado em:11 de setembro 2019.

DAY, R. Deleuze e Guattari e a Psicologia Cognitiva, IA e IHC: investigando possíveis conexões e diferenças. InCID: Revista de Ciencia da Informação e Documentação., V.1. $\mathrm{N}^{\mathrm{o}}$.2. 2010. P.3-18. Disponível em: http://www.revistas.usp.br/incid/article/view/42316 Consultado em:11 de setembro 2019.

DODEBEI, V. Cultura Digital: novo sentido e significado de documento para a memória social? DataGramaZero - Revista de Ciência da Informação.V.12. N N$^{\circ}$.2. 2011. Disponível em: https://es.scribd.com/document/86362798/DODEBEI-V-Cultura-Digital-novo-sentido-esignificado-de-documento-para-a-memoria-social Consultado em: 11 de setembro 2019.

FLORIDI, L. What Is the Philosophy of Information? Metaphilosophy, V.33. No1-2. 2002. P.123-145. Disponível em:

https://www.academia.edu/3491627/What_is_the_Philosophy_of_Information Consultado em:11 de setembro 2019.

GIBSON, K., LADD, M., PRESNELL, J. Traversing the Gap. Subject Specialists Connecting Humanities Researchers and Digital Scholarship Centers. In Digital Humanities in the library: Challenges and Opportunities for Subject Specialists. EUA: The Association of College \& Research Libraries. 2015. P. 3-17. Disponível em: https:/sc.lib.miamioh.edu/bitstream/handle/2374.MIA/5218/Digital\%20Humanities\%20Chap ter\%201.pdf?sequence=1\&isAllowed=y Consultado em: 11 de setembro 2019 .

KOLTAY, T. Library and information science and the digital humanities: Perceived and real strengths and weaknesses. Journal of Documentation, V.72. N.4. 2016. P.781-792. 
Disponível em: https://doi.org/10.1108/JDOC-01-2016-0008 Consultado em: 11 de setembro 2019.

KOLTAY, T. Library and information science and the digital humanities: are they sciences of the information? Informacios Tarsadalom, V.13. №2. 2017.

LATOUR, B. Ciencia en acción. Barcelona: Labor, 1992.

ORTEGA, E., GUTIÉRREZ, S. MapaHD. Una exploración de las Humanidades Digitales en español y portugués. In: Cuadernos Artesanos de Comunicación: Ciencias Sociales y Humanidades Digitales. Técnicas, herramientas e-Research e investigación en colaboración. Tenerife, España: Sociedad Latina de Comunicación Social. Vol. 61. 2014. Disponível em: https://www.researchgate.net/publication/303985389_MapaHD_Una_exploracion_de_las_Hu manidades_Digitales_en_espanol_y_portugues Consultado em: 11 de setembro 2019.

POSNER, M. Digital Humanities and the Library. Journal of Library Administration, V.53. $N^{\circ}$.1. 2013. Disponível em: http://miriamposner.com/blog/digital-humanities-and-thelibrary/ Consultado em: 11 de setembro 2019.

RAMSAY, S. Care of the soul. Lecture conducted from Emory University. 2010. Disponível em: http://stephenramsay.us/text/2010/10/08/care-of-thesoul.htm Consultado em: $11 \mathrm{de}$ setembro 2019.

ROBINSON, L., PRIEGO, E., BAWDEN, D. Library and Information Science and Digital Humanities: Two Disciplines, Joint Future? 14th International Symposium on Information Science, Zadar, Croatia. 2015. Disponível em: http://openaccess.city.ac.uk/11889/ Consultado em: 11 de setembro 2019.

SCHREIBMAN, S., SIEMENS, R., UNSWORTH, J. (Eds.). A Companion to Digital Humanities. 2004. Disponível em: http://www.digitalhumanities.org/companion/ Consultado em: 11 de setembro 2019.

SPIRO, L. Computing and communicating knowledge: collaborative approaches to digital humanities projects. In: Collaborative Approaches to the Digital in English Studies. 2011. P. 44-81. Disponível em: https://ccdigitalpress.org/book/cad/Ch2_Spiro.pdf Consultado em: 11 de setembro 2019.

SULA, C. A. Digital Humanities and Libraries: A Conceptual Model. Journal of Library Administration, V.53. No1. 2013. P.10-26. Disponível em: https://doi.org/10.1080/01930826.2013.756680177 Consultado em: 11 de setembro 2019.

VANDEGRIFT, M., VARNER, S. Evolving in Common: Creating Mutually Supportive Relationships Between Libraries and the Digital Humanities. Journal of Library Administration, V.53. №1. Disponível em: https://doi.org/10.1080/01930826.2013.756699 Consultado em: 11 de setembro 2019. 
VINCK, D. Las culturas y humanidades digitales como nuevo desafío para el desarrollo de la ciencia y la tecnología en América Latina. V.22. 2013. Disponível em:

http://www.scielo.org.co/pdf/unih/n76/n76a04.pdf Consultado em: 11 de setembro 2019.

ZHANG, Y., LIU, S., MATHEWS, E. Convergence of digital humanities and digital libraries. Library Management, V.36. No ${ }^{\circ} 4 / 5,2015$. P.362-377. Disponível em:

https://www.researchgate.net/publication/277940906_Convergence_of_digital_humanities_an d_digital_libraries Consultado em: 11 de setembro 2019 . 